Original Research Paper

\title{
The Optimization of a Porous Ti6Al4V Bone Construct Using Additive Manufacturing
}

\author{
${ }^{1}$ Glenn Sanders, ${ }^{1}$ Jeffrey Wilk, ${ }^{1}$ Shelby Marks, \\ ${ }^{1}$ S. Alex Paolicelli, ${ }^{2}$ Matthew DiCaprio and ${ }^{1}$ Ronald Bucinell \\ ${ }^{1}$ Department Mechanical Engineering, Union College, Schenectady, NY, USA \\ ${ }^{2}$ Department of Orthopaedic Surgery, Albany Medical Center, Albany, NY, USA
}

Article history

Received: 01-12-2016

Revised: $14-12-2016$

Accepted: 14-12-2016

Corresponding Author:

Glenn Sanders

Department Mechanical

Engineering, Union College,

Schenectady, NY, USA

Email: gsanders12@gmail.com

\begin{abstract}
Patient specific implants are becoming viable treatment options in some orthopaedic applications through advances in additive manufacturing and $3 \mathrm{D}$ printing techniques. One potential application is for treatment of segmental bone defects, particularly in patients suffering from bone cancer. Current treatment options are: Amputation, megaprosthesis, or allografts. These treatments are often highly invasive, may require a partial/full joint replacement and are limited by mechanical properties, which affect the life of the implant. The Ti6Al4V implant proposed in this research was designed to fit a mid-diaphyseal segmental bone defect, mimic the mechanical properties of bone, facilitate osseointegration and reduce wear at the bone-implant surface. Computer-Aided Designs (CAD) were constructed of patient-specific Ti6Al4V implants based off the geometry of (1) a patient suffering from a lesion on the mid-diaphysis of the femur and, (2) a 4th Generation right Sawbones ${ }^{\circledR}$ femur. Pore size and shape were assessed using Finite Element Analyses (FEA) software. The overall porosity was maximized to develop an implant with an effective elastic modulus equivalent to bone. The two implants were then fabricated using Direct Metal Laser Sintering (DMLS). The geometry of the physical implant was measured and mechanically loaded under compression to validate the computational model. FEA was an effective tool for optimizing the pore size, shape and overall porosity of the implant, which indicated that $1 \mathrm{~mm}$ circular pores in three orthogonal planes at an overall porosity of $54-76 \%$ would produce an implant with an effective elastic modulus equivalent to cortical bone. Geometric analysis of the 3D printed implant indicated the pore sizes were reduced by an average of $16 \%$ as compared to the computational model and that there was a correlation between the size and precision of the pore and the orientation of the implant during the additive build. Compression testing of the implants indicated that they had an effective elastic modulus of 20.8 and $10.5 \mathrm{GPa}$, which is within the accepted values for cortical bone.
\end{abstract}

Keywords: Three-Dimensional Printing, Bone Scaffold, Porous, Titanium, Tissue Engineering, Osteosarcoma

\section{Introduction}

Osteosarcoma is the most common primary bone malignancy found in children. The most common skeletal sites of disease include the distal femur, proximal tibia and proximal humerus. Lesions frequently affect the metaphyseal or diaphyseal segment of the bone (Vander, 1996). There is a bimodal peak in incidence with the majority occurring during adolescence (10-14 years) and in elderly patients (over 65 years) (Ottaviani and Jaffe, 2010). Osteosarcoma presents at a rate of 5.4 per million per year in males and 4.0 per million per year in females (Vander, 1996). 42\% of all osteosarcoma cases originate within the femur, with $75 \%$ of tumors occurring in the distal metadiaphyseal region (Ries et al., 1999). The five-year survival rate for osteosarcoma patients is $68 \%$ (Vander, 1996; DiCaprio and Friedlaender, 2003; Gibbs et al., 2001). 
Osteosarcoma can result in severe repercussions for patient mobility and quality of life. Due to the aggressive nature of the tumor, the identification of the cancerous tissue and subsequent treatment is crucial for patient survival. The primary goal of the treatment is to remove the diseased section of bone and to secondarily restore the functionality of the patient. This can be achieved through amputation or by removing the local section of diseased bone. When only the local bone is removed, a segmental bone defect is left. In order to restore functionality, this defect must be filled. Current treatment methods include: Amputations, allograft insertion and megaprostheses. Amputations involve the complete resection of the affected limb. Allografts are bone-transplants from cadaveric tissue. Megaprostheses are large implants that span the defect and also incorporate a total joint replacement. These treatments are limited in scope and can have detrimental effects for the post-operative prognosis.

Amputations have severe repercussions for the patient's quality of life, such as discomfort, infection, phantom pain, skin irritation, mobility and expense (Yang, 2004). Limb saving procedures, such as allograft/megaprosthesis insertion and radiotherapy methods have reduced the need for amputation, yet present additional problems and have not been shown to increase long term patient survival (Simon et al., 1986).

Allograft implantation is a less invasive method, but is plagued by intangible factors such as: Inadequate curettage of a bone cyst, inconsistent stabilization with polymethylmethacrylate, subsequent cellular degeneration through radiotherapy and immunorejection (Friedlaender, 1991). Rejection occurs through antibodymediated cytoxicity, similar to organ donor rejection (Aro and Aho, 1993). In some advanced osteosarcoma cases, the osteogenic ability of bone is impaired, leading to delayed union and longer healing times and ultimately failure (Gómez-Barrena et al., 2015).

Megaprosthesis procedures are highly invasive, require a full distal or proximal joint replacement and can cause complications in adolescents whose bones have not matured fully (Ottaviani and Jaffe, 2010). Megaprostheses replace an anatomical joint and/or segment of bone and through cyclical loading are subject to fatigue and subsequent mechanical failure and wear about certain soft tissues (Heisel et al., 2006; Chuang et al., 2013). Medical intervention remains the most definitive and successful path for treatment of osteosarcoma (Ottaviani et al., 2009).

Advances in additive manufacturing techniques can improve upon the shortcomings of current treatments through the use of custom patient specific implants. Patient specific implants reduce problems associated with standardized surgical implant production, including imperfect fit, wear along the bone-implant surface and post-operative pain and mobility (Choong et al., 2009).
Additive manufactured implants can be functionalized at an engineer's discretion; CAD models can be created containing geometries and surface characteristics which promote osseointegration, then be fabricated through 3D printed implants.

DMLS is a powder sintering method that fuses metal powders together through focused melting and quenching with a laser beam. A unique advantage to this fabrication technique over conventional machining is the material selection and precision of the process. Several different biocompatible materials can be used depending on the printed device's function. For medical applications, Ti6Al4V is a commonly used alloy. Ti6Al4V is favored for its corrosion resistance, low weight and biocompatibility (Sedlák et al., 2013).

Titanium has two distinct allotropic forms. The $\alpha$ phase of titanium exists up to a transition point at $882^{\circ} \mathrm{C}\left(1620^{\circ} \mathrm{F}\right)$ in a hexagonal lattice. Above the transformation point, the $\beta$ phase of titanium take the form of a body centered cubic lattice. $\alpha$-stabilizing interstitial elements include carbon, oxygen, nitrogen and aluminum. $\beta$-Stabilizing elements include vanadium, molybdenum, chromium, iron and manganese. Ti6Al4V is used for its $\alpha+\beta$ phase stability, which occurs due to its high percentage of aluminum and vanadium interstitial substitutions, which give it a balanced mix of mechanical properties and a high fatigue life, well suited for the medical field.

The purpose of this research was to optimize the design, fixation and fabrication of a patient-specific alloy femoral implant, while facilitating osseointegration. Osseointegration is the process of bone growth onto and into the implant and correlates with the long-term survival of the implant. Implantation without optimizing osseointegration could possibly lead to a reduction in the mechanical properties of the bone tissue adjacent to the implant site, resulting in a compromised femoral integrity. The implant was designed to mimic the mechanical properties of the cortical and cancellous components of the patient's femur. The method of simulating bone tissue was to decrease the effective modulus of the titanium implant to the modulus of the surrounding bone tissue. Geometric modifications were made to the modeled implant in order to reduce the effective elastic modulus of the device and promote osseointegration. Characterization of this behavior through finite element analysis and, subsequently, mechanical loading will provide insight into how the implant would mechanically behave in vivo while also understanding the predictive capabilities of the finite element analysis and the limitations of the DMLS process.

\section{Materials and Methods}

\section{Implant Design}

Computed Tomography (CT) scans were taken of a patient containing a mid-diaphyseal lesion in the left 
femur. CT data were brought into medical imaging software (Materialise, Leuven, Belgium) and segmentation was applied to isolate bony tissue (Fig. 1a). The femur was then reconstructed three-dimensionally (Fig. 1b) and a "virtual surgery" was conducted to remove the diseased tissue including appropriate proximal and distal margins (Fig. 1c). The femur, including the segmental bone defect, was then brought into CAD (SolidWorks, Waltham, MA, USA) and a patient-specific implant was designed to fit the defect (Fig. 1d and 1e).

\section{Pore Optimization}

Finite element analyses (SolidWorks Simulation, Waltham, MA, USA) was used to assess the efficacy of pore shape, pore orientation and overall porosity on a Ti6Al4V implant under physiologic load magnitudes. In all studies, titanium alloy (Ti6Al4V) properties were applied to the implant, the distal portion of the implant was fixed in plane and an axial force of $1800 \mathrm{~N}$ was applied. The implant was automeshed using tetrahedral elements. Peak stresses and the effective elastic moduli were measured for implants using three different pore shapes: Circular, square and triangular. Each pore had equal crosssectional area $\left(0.785 \mathrm{~mm}^{2}\right)$ and each implant had equal pore density (100 axial pores). Three different pore orientations were also assessed: Axial only, transverse only and combined axial and transverse in three orthogonal directions. Overall stress distributions were also characterized. Pore density was then increased until the modulus of the implant reached that of cortical bone (10-21 GPa) (Rho et al., 1993).

\section{Experimental Validation}

Based on the optimized computational design, two implants were fabricated using DMLS. The first generation (Gen 1) implant was designed to fit the specific patient obtained in the CT scan. The second generation (Gen 2) implant was designed to fit a synthetic femur (4th Generation SawBones, Pacific Research Laboratories, Vashon Island, WA). Mass and bulk volume measurements of the fabricated implant were taken and compared to the predicted values from the computational model. Scanning Electron Microscopy (SEM) was used to characterize the pore diameters in the transverse plane. Due to size constraints, pores in the axial direction were measured using an optical microscope. Fifteen pores in each plane were characterized and ANOVA was used to test for significant difference in pore diameters amongst the three planes. Following geometric measurements, axial compression was applied (Instru-Met Corporation, Union, NJ) to the implant at a rate of 0.01 inches $/ \mathrm{min}$. Implants were loaded to $600 \mathrm{~N}$ and $1200 \mathrm{~N}$. Four data sets for each force magnitude were collected and the average effective elastic modulus was used to compare to computational models. The effective elastic modulus is defined using Equation 1:

$$
E_{\text {eff }}=\sigma / \varepsilon
$$

Where:

$$
\begin{array}{ll}
E_{\text {eff }} & =\text { Effective elastic modulus } \\
\sigma & =\text { Applied stress } \\
\varepsilon & =\text { Measured strain }
\end{array}
$$

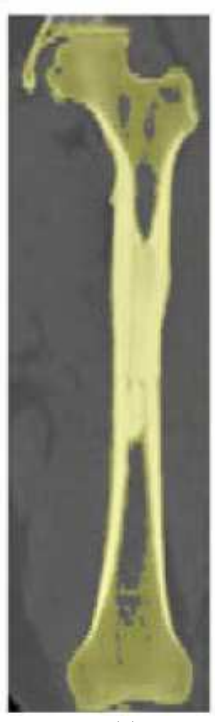

(a)

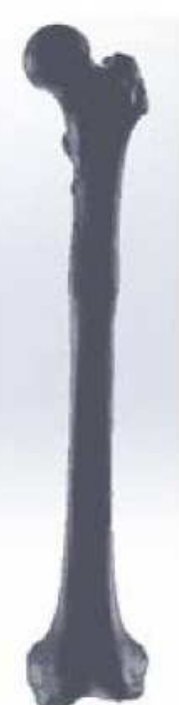

(b)

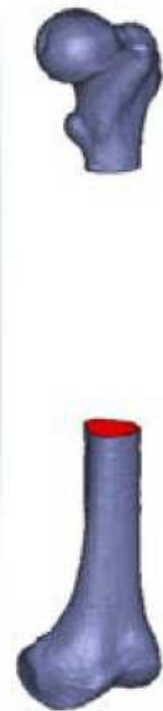

(c)

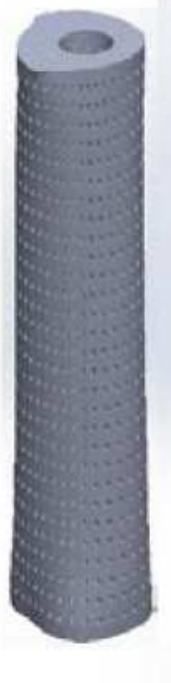

(d)

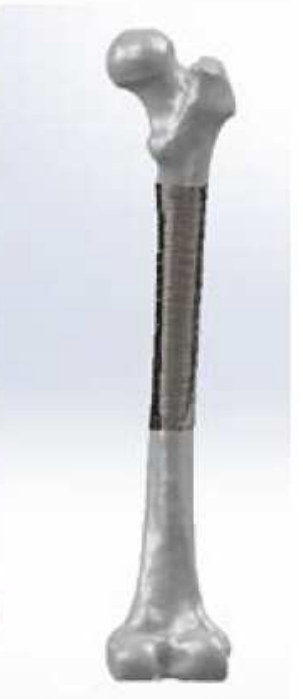

(e)

Fig. 1. This figure shows the implant design process from segmenting the bony tissue in the CT data (a), the $3 \mathrm{D}$ reconstruction of the diseased femur (b), the virtual surgery and tissue resection (c), the design of the porous implant (d) and the implant femur (e) 


\section{Results}

\section{Implant Design}

The diseased bone of the femur was successfully isolated by applying a lower threshold of 73 Hounsfield Units and an upper threshold of 1762 Hounsfield Units to segment the bone from the adjacent soft tissue. The virtual surgery resulted in a $15 \mathrm{~cm}$ segmental bone defect. The superior margin of the defect was located $8 \mathrm{~cm}$ from the greater trochanter of the femur. For proofof-concept, the size of the implant studied in this experiment was reduced to $5.6 \mathrm{~cm}$ in height.

\section{Pore Optimization}

The addition of circular, square and triangular pores of equivalent cross-sectional area reduced the effective elastic modulus from the inherent modulus for Ti6Al4V, which is $120 \mathrm{GPa}$, to $98.0,98.2,98.5 \mathrm{GPa}$, respectively. However, stress concentrations around the edges of the square and triangular pores were $59 \%$ higher than the stress concentrations around the circular pores. Axial,

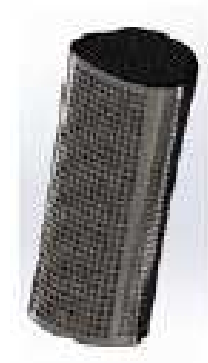

(a)

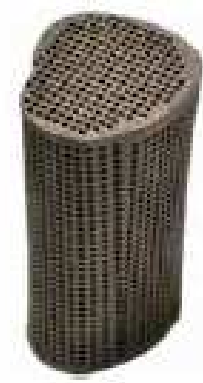

(b)

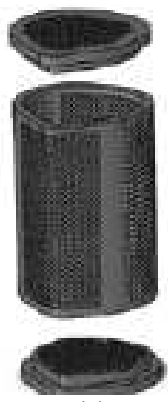

(c)

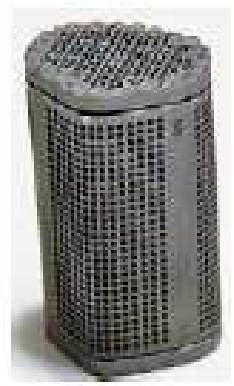

(d)

Fig. 3. Computational model and photograph of the Gen 1 patient-specific implant (a,b) and the Gen 2 patient-specific implant (c,d)

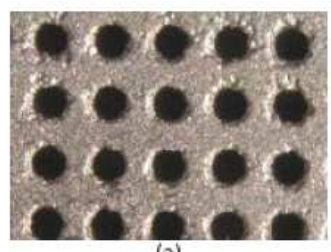

(a)

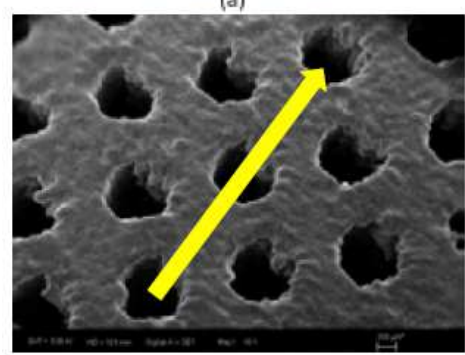

(b)

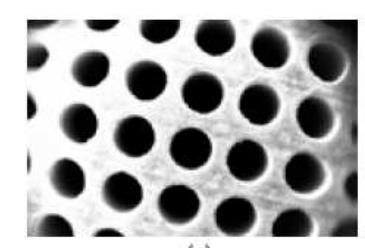

(c)

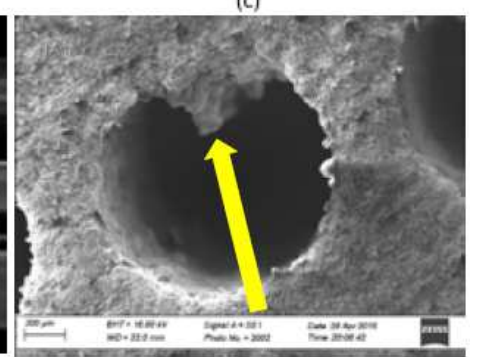

(d)

Fig. 4. An optical microscope image of axial pores in the Gen 1 implant (a), SEM image of transverse pores in the Gen 1 implant (b), optical microscope image of axial pores in the Gen 2 implant (c) and SEM image of transverse pores in the Gen 2 implant (d) shows increased centricity in axial pores. Yellow arrows indicate the build direction of the implant which correlates with a deposit of material at the top of each pore 

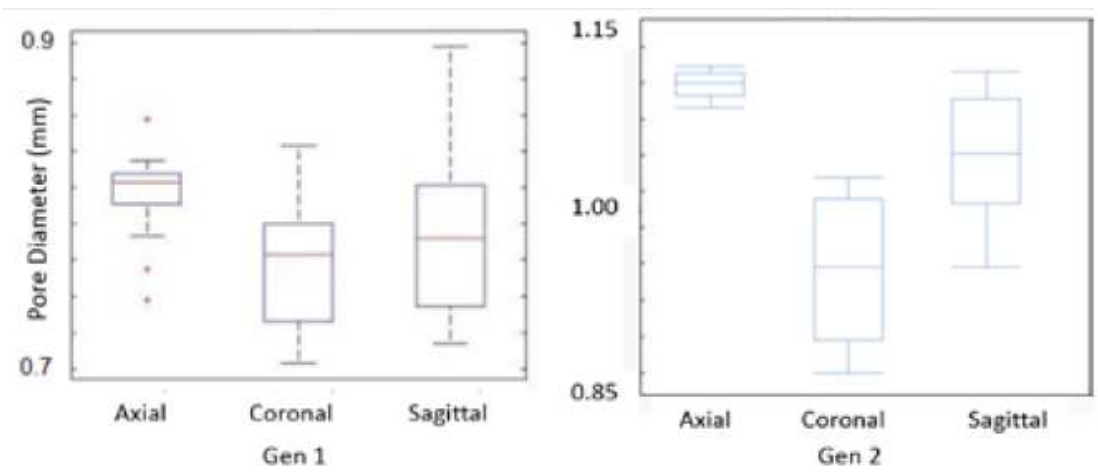

Fig. 5. A box-and-whisker plot of pore diameter measurements indicate that axial pores were larger than transverse pores and were more precise, which correlated with the primary build direction of the implant. Similar trends were seen in both Gen 1 and Gen

Table 1. Computational implant design

\begin{tabular}{ll}
\hline Gen 1 implant & Gen 2 implant \\
\hline $5.6 \mathrm{~cm}$ height & $5.0 \mathrm{~cm}$ height \\
$\sim 3.0 \mathrm{~cm}$ average OD & $\sim 3.16 \mathrm{~cm}$ average OD \\
$\sim 1.6 \mathrm{~cm}$ average intramedullary cavity & $\sim 2.6 \mathrm{~cm}$ average intramedullary cavity \\
$1 \mathrm{~mm}$ pores with $1.6 \mathrm{~mm}$ spacing-orthogonal directions & $1.19 \mathrm{~mm}$ pores with $1.5 \mathrm{~mm}$ spacing-orthogonal directions \\
$54 \%$ porosity & $73 \%$ porosity \\
$\mathrm{E}=17.6 \mathrm{GPa}$ & $\mathrm{E}=13.6 \mathrm{GPa}$ \\
$1.5 \mathrm{~mm}$ fixed endplates on the top and bottom. & $2.5 \mathrm{~mm}$ removable endplates on the top and bottom. \\
\hline
\end{tabular}

Table 2. Computational and physical mass and volume measurements of the Gen 1 and Gen 2 IMPLANT

\begin{tabular}{llllr}
\hline & & $\begin{array}{l}\text { Computational } \\
\text { prediction }\end{array}$ & $\begin{array}{l}\text { Physical } \\
\text { measurement }\end{array}$ & $\begin{array}{c}\% \\
\text { difference }\end{array}$ \\
\hline \multirow{3}{*}{ Gen 1} & Mass $(\mathrm{g})$ & 59.20 & 59.60 & 0.7 \\
& Volume $\left(\mathrm{cm}^{3}\right)$ & 13.37 & 16.00 & 19.7 \\
Gen 2 & Mass $(\mathrm{g})$ & 46.70 & 47.90 & 2.5 \\
\hline
\end{tabular}

Based on these conclusions, two implants were designed with the properties shown in Table 1 . Computational models and manufactured implants can be seen in Fig. 3.

A comparison between the computational model and physical implant for mass and volume measurements is shown in Table 2. Optical microscope and SEM images of axial and transverse pores are shown in Fig. 4. Transverse pores were more eccentric than axial pores in both implants. Transverse pores also contained a deposit of material that correlated with the top of each pore in the direction that the implant was printed.

SEM and optical microscopy revealed that the average pore diameter of the DMLS implants were 16\% less than the computational model (Fig. 5). It was also observed that pores in the transverse plane were smaller than those in the axial plane $(\mathrm{p}<0.05)$.

Static compression indicated an effective elastic modulus of 20.8 and $10.5 \mathrm{GPa}$ for the Gen 1 and Gen 2 implants, respectively. Predicted moduli for the Gen 1 and Gen 2 implant were 17.6 and $13.6 \mathrm{GPa}$, respectively.

\section{Discussion}

We have demonstrated a process for designing a patient-specific scaffold to treat bone cancers and other large segmental bone defects. CT data of a diseased femur was successfully used to design a patient-specific implant to fit a segmental bone defect.

Computational analyses showed that axially applied pores were more effective at lowering the elastic modulus then transversely applied pores; although, a combination of both patterns was most effective and was necessary to reduce the modulus of the titanium implant to that of bone. Computational analyses also showed that varying pore shape had a minimal effect on reducing the effective elastic modulus. This is expected according to the governing mechanics formulas (Equation 2). As noted by this relationship, if the cross-sectional area of each pore remained constant, the deflection of the construct will remain constant:

$\delta=P L / A E$ 
Where:

$\delta=$ Deflection

$P=$ Applied force

$L=$ Length of implant

$A=$ Cross-sectional area

$E=$ Modulus

Because the square and triangular-shaped pores resulted in a $59 \%$ increase in stress magnitudes as compared to circular pores, circular pores were chosen so that the overall porosity could be maximized without jeopardizing the mechanical strength of the implant. Overall porosities between 54 and $76 \%$ were found to result in an effective elastic modulus equivalent to cortical bone. It is expected that a modulus of 10-21 GPa will result in improved loadsharing and an optimal environment for osseointegration (Choong et al., 2009).

The ability for DMLS to create complex geometries out of a highly resistant material is prized throughout the medical community and was paramount to the manufacturing aspects of this study. The microporosity of the aggregate fused powders has been shown to promote an osseoconductive environment (Ottaviani and Jaffe, 2010). Coupled with the biocompatible properties of the titanium itself, DMLS will be crucial for the development of complex custom metallic implants, specifically where osseointegration and mechanical stability is the long-term goal.

This study showed that DMLS may lead to a $16 \%$ reduction in pore size as compared to the computational design. We also showed that imperfections in geometry may occur. Because additive manufacturing builds volumes layer by layer, there appears to be a correlation with the imperfections and the build direction of the implant. These differences should be taken into account when designing implants.

Using DMLS, the porosity and pore size of these implants can be finely tuned to improve load sharing and optimize osseointegration. Using this additive manufacturing method, the implant was fabricated out of titanium alloy, resulting in an implant with improved mechanical properties as compared to traditional allograft. The ultimate strength of allografts ranges from $0.15-30 \mathrm{MPa}$, while the ultimate strength of Ti6Al4V is approximately 1000 $\mathrm{MPa}$ (Ries et al., 1999), indicating that the porous titanium alloy implant will have a greater fatigue life than allografts. The porous structure of the titanium implant may also result in improved vascularity and nutrition, resulting in improved osseointegration.

Using these manufacturing techniques, the porous implant is less invasive than traditional megaprostheses surgical techniques and also have the invaluable benefit of sparing the patient's joint. FEA and experimental results reveal that the stiffness can be reduced to minimize stress-shielding and that peak stresses remain well below the endurance limit.

Limitations of this study include a small sample size. As such, the effects of build direction and various postprocessing techniques aimed at improving mechanical properties of the DMLS implant were beyond the scope of the reported study. Also, only a simplified loading scenario of static compression was analyzed experimentally. In actuality, most loading scenarios in the femur is complex and results in a combination of compression and bending. Future studies should be aimed at increasing the sample size and studying more complex loading scenarios.

We believe that additive manufacturing will become a useful tool in treating not only bone cancer patients, but other large bone segmental defects and orthopaedic conditions, such as high energy fractures. Incorporation of elements used within this study, such as CT scanning to DMLS printing may provide an alternative to current invasive treatment methods used to treat bone defects.

\section{Conclusion}

Additive manufacturing has recently gained a large amount of excitement in the orthopaedic field for a wide variety of disorders, which include segmental bone defects to treat bone cancer as well as total joint arthroplasty. Before these implants can be inserted, it is critical to understand constraints with the manufacturing methods. In this study, we propose a novel method for treating segmental bone defects that occur with conditions such as bone cancer. We conducted initial implant design and mechanical testing on a porous Ti6Al4V implant. Results indicate that FEA is a useful tool for predicting the mechanical properties of the porous implant. Mechanical testing validated the computational models, which resulted in a porous titanium implant that had an effective elastic modulus equivalent to cortical bone. This study shows some of the constraints associated with additive manufacturing and ultimately concludes that additive manufacturing and particularly DMLS, remains a promising method of fabrication for titanium alloy orthopaedic implants.

\section{Acknowledgement}

Funding for this research was provided by Union College in the form of an internal faculty research grant. Technical support was provided by Stanley Gorski, Rebecca Cortez, Andrew Rapoff and Scott Kirkton.

\section{Funding Information}

This research was funded by a faculty research grant at Union College. 


\section{Author's Contributions}

Glenn Sanders: Conducted design, mechanical analyses and management of the research efforts.

Jeff Wilk, Shelby Marks and S. Alex Paolicelli: Conducted design and mechanical analyses throughout the study.

Ronald Bucinell: Conducted mechanical analyses and provided mechanical testing guidance.

Matthew DiCaprio: Provided clinical guidance and expertise in osteosarcoma treatments.

\section{Conflicts of Interest}

None

\section{References}

Aro, H.T. and A.J. Aho, 1993. Clinical use of bone allografts. J. Ann. Med., 25: 403-412.

DOI: $10.3109 / 07853899309147303$

Choong, P.F., M.M. Dowsey and J.D. Stoney, 2009. Does accurate anatomical alignment result in better function and quality of life? Comparing conventional and computer-assisted total knee arthroplasty. J. Arthroplasty, 24: 560-569.

DOI: 10.1016/j.arth.2008.02.018

Chuang, M.Y., T.K. Chang, C.H. Huang and T.Y. Huang, 2013. Failure of the rotating-hinge knee megaprosthesis. J. Arthroplasty, 543: e5-e8. DOI: 10.1016/j.arth.2012.07.030

DiCaprio, M.R. and G.E. Friedlaender, 2003. Malignant bone tumors: Limb sparing versus amputation. J. Am. Acad. Orthop. Surg., 11: 25-37. PMID: 12699369

Friedlaender, G.E., 1991. Bone allografts: The biological consequences of immunological events. J. Bone Joint Surg. Am., 73: 1119-1122.

Gibbs, C.P.J., K. Weber and M.T. Scarborough, 2001. Malignant bone tumors. J. Bone Joint Surg., 83: $1728-1745$.

Gómez-Barrena, E., P. Rosset, D. Lozano, J. Stanovici and C. Ermthaller et al., 2015. Bone fracture healing: Cell therapy in delayed unions and nonunions. Bone, 70: 93-101. DOI: $10.1016 /$ j.bone.2014.07.033

Heisel, C., S. Kinkel and L. Bernd, 2006. Megaprostheses for the treatment of malignant bone tumours of the lower limbs. Int. Orthop., 30: 452-457. DOI: $10.1007 / \mathrm{s} 00264-006-0207-7$

Sedlák, J., M. Ptácková, J. Nejedly, M. Madaj and J. Dvorácek et al., 2013. Material analysis of titanium alloy produced by direct metal laser sintering. Int. J. Metalcast., 7: 43-50. DOI: 10.1007/BF03355552
Ottaviani, G. and N. Jaffe, 2010. The Epidemiology of Osteosarcoma. In: Pediatric and Adolescent Osteosarcoma, Jaffe, N., O.S. Bruland and S. Bielack (Eds.), Springer US, Boston, ISBN: 10- 978-1-4419-0284-9, pp: 3-13.

Ottaviani, G., R.S. Robert, W.W. Huh and N. Jaffe, 2009. Functional, psychosocial and professional outcomes in long-term survivors of lower-extremity osteosarcomas: Amputation versus limb salvage. Cancer Treat Res., 152: 421-436. DOI: 10.1007/978-1-4419-0284-9_23

Rho, J.Y., R.B. Ashman and C.H. Turner, 1993. Young's modulus of trabecular and cortical bone material: Ultrasonic and microtensile measurements. J. Biomechan., 26: 111-119. DOI: 10.1016/0021-9290(93)90042-D

Ries, L.A.G., M.A. Smith and J.G. Gurney, 1999. Cancer incidence and survival among children and adolescents. United States SEER Program 1975-1995.

Simon, M.A., M.A. Aschliman, N. Thomas and H.J. Mankin, 1986. Limb-salvage treatment versus amputation for osteosarcoma of the distal end of the femur. J. Bone Joint Surg. Am., 87: 2822. DOI: $10.2106 / J B J S .8712 . c l$

Vander, G.R.A., 1996. Osteosarcoma and its variants. Orthop. Clin. North Am., 27: 575-581. PMID: 8649738

Yang, R.S., 2004. Endoprosthesis-related complications after limb-salvage operation of malignant bone tumors around the knee. Biomed. Eng. Appl. Basis Commun., 16: 133-142.

DOI: $10.4015 / \mathrm{S} 1016237204000189$ 